\title{
Letter
}

\section{On MHD stability of gravitating electrically conducting fluids with field-aligned flows}

\author{
H. T A S S ${ }^{1}$ and G. N. THR O U M O U L P O U L O S \\ ${ }^{1}$ Max-Planck-Institut für Plasmaphysik, Euratom Association, 85748 Garching bei München, Germany (het@ipp.mpg.de) \\ ${ }^{2}$ Department of Physics, University of Ioannina, Association Euratom-Hellenic Republic, GR 45110 Ioannina, Greece \\ (gthroum@uoi.gr)
}

(Received 3 November 2009; revised 29 November 2009; accepted 15 July 2011; first published online 18 August 2011)

\begin{abstract}
A previous stability condition (see Throumoulopoulos and Tasso, Phys. Plasmas 14, 122104 (2007)) for incompressible plasmas with field-aligned flows is extended to gravitating plasmas, including self-gravitation. It turns out that the stability condition is affected by gravitation through the equilibrium values only.
\end{abstract}

In a previous paper [1] a sufficient condition was derived for the linear stability of plasmas of uniform density and incompressible flow parallel to the magnetic field. This condition states that a generic steady state of the plasma is stable to three-dimensional perturbations if the flow is sub-Alfvénic $\left(\lambda^{2}<1\right.$, where $\lambda$ is the Alfvén Mach function) and $A \geqslant 0$ with

$$
\begin{aligned}
A= & -g^{2}\left[\left(1-\lambda^{2}\right)(\mathbf{J} \times \nabla \psi) \cdot(\mathbf{B} \cdot \nabla) \nabla \psi\right. \\
& \left.+\frac{\left(\lambda^{2}\right)^{\prime}}{2}|\nabla \psi|^{2}\left(\nabla \psi \cdot \nabla \frac{B^{2}}{2}+g|\nabla \psi|^{2}\right)\right], \\
g\left(\psi, B^{2}\right)= & \frac{P_{s}^{\prime}}{1-\lambda^{2}}-\frac{\left(\lambda^{2}\right)^{\prime}}{1-\lambda^{2}} \frac{B^{2}}{2} .
\end{aligned}
$$

Here, $\psi$ is a smooth function which labels the magnetic surfaces, $\mathbf{B}$ is the magnetic field, $B$ is the magnetic field modulus, $\mathbf{J}=\nabla \times \mathbf{B}$ is the current density, the surface function $P_{S}(\psi)$ coincides with the thermal pressure when the flow vanishes and the prime indicates a derivative with respect to $\psi$. All the quantities in (1) and (2) pertain to equilibrium. Aim of the present note is to extend the aforementioned condition to gravitating plasmas.

As in [1], the system under consideration is a constant density plasma with an incompressible flow aligned with B, which, in addition to Lorenz and thermal pressure forces, is maintained in a magnetohydrodynamic equilibrium by external and self-gravitation. It is governed by the following equations:

$$
\mathbf{v}=\lambda \mathbf{B}
$$

with $\lambda$ being an arbitrary function,

$$
\nabla \cdot \mathbf{v}=0
$$

$$
\mathbf{v} \cdot \nabla \mathbf{v}=\mathbf{J} \times \mathbf{B}-\nabla P+\nabla \phi,
$$

where $\mathbf{v}$ is the velocity, $P$ is the thermal pressure and $\phi$ is the gravitational potential. The equations are written in convenient units and the constant density is set to 1 . In fact, due to incompressibility it suffices to assume constant density only at equilibrium. Also, we assume the existence of well-defined equilibrium magnetic surfaces.

Equations (3) and (5) lead to $\lambda=\lambda(\psi)$, and using the identity $\mathbf{v} \cdot \nabla \mathbf{v}=\nabla v^{2} / 2-\mathbf{v} \times \nabla \times \mathbf{v}$, (3)-(5) lead to

$$
\left(1-\lambda^{2}\right) \mathbf{J} \times \mathbf{B}=\nabla\left(P+\frac{\lambda^{2} B^{2}}{2}-\phi\right)-B^{2} \nabla \frac{\lambda^{2}}{2},
$$

where $v$ is the velocity field modulus. Taking the scalar product of (6) with $\mathbf{B}$ gives

$$
P+\frac{\lambda^{2} B^{2}}{2}-\phi=P_{s \phi}(\psi)
$$

where $P_{s \phi}(\psi)$ is the arbitrary function in the presence of gravity.

Consequently, (6) can be put in the form

$$
\left(1-\lambda^{2}\right) \mathbf{J} \times \mathbf{B}=P_{s \phi}^{\prime} \nabla \psi-\left(\lambda^{2}\right)^{\prime} \frac{B^{2}}{2} \nabla \psi,
$$

with

$$
\mathbf{J} \times \mathbf{B}=g_{\phi}\left(\psi, B^{2}\right) \nabla \psi
$$

where

$$
g_{\phi}\left(\psi, B^{2}\right)=\frac{P_{s \phi}^{\prime}}{1-\lambda^{2}}-\frac{\left(\lambda^{2}\right)^{\prime}}{1-\lambda^{2}} \frac{B^{2}}{2} .
$$

The only difference with [1] is the presence of the gravitational potential $\phi$ in (5)-(10). This potential obeys Poisson's equation

$$
\Delta \phi=-1,
$$

whose solution is the superposition of the internal potential $\phi_{i}$ given by $\phi_{i}=\int_{V} \frac{1}{\left|\left(\mathbf{x}-\mathbf{x}^{\prime}\right)\right|} d^{3} \mathbf{x}^{\prime}$, where $V$ is the volume 
of the considered plasma, and an external potential $\phi_{e}$ due to fixed masses outside the plasma.

Let us now come to the stability of our flowing and gravitating equilibria. The general problem without gravity has been already formulated in [2] in terms of the Lagrangian displacement $\xi$. Its application to equilibria with field-aligned incompressible flows and homogeneous density was investigated in $[1,3]$. The correct derivation of the final condition briefly presented here in the preamble was done in [1]. Now note that the quantity $g$ in (2) is like the $g_{\phi}$ in (10), but $P_{s}$ in it does not contain the gravitational potential $\phi$. Therefore, $A \geqslant 0$ remains valid as a stability criterion if $g$ in (1) is replaced by $g_{\phi}$. The reason is that the perturbed density, $\rho_{1}$, in the linearized dynamical equations vanishes for $\nabla \cdot \xi=0$ and homogeneous density at equilibrium. Consequently, it follows that $\phi_{1}=0$, as the internal potential is determined solely by the plasma density distribution and the external potential, by definition, is unaffected by the perturbation. Hence, $\rho \nabla \phi_{1}=0$ and the perturbed momentum equation has the same form with and without the presence of gravity. The fact that for uniform equilibrium density, the density remains uniform in the perturbed state because of incompressibility was not noticed in $[1,3]$.

We conclude by stating that the above-mentioned condition is sufficient for stability if we replace $g$ in (1) by $g_{\phi}$ [Eq. (10)], which means that this stability condition is affected by gravity through the equilibrium values only. A possible application of the condition in the framework of an idealized model could be the stability of the earth magma. It is emphasized, however, that the condition is applicable to internal modes of generic plasma equilibria.

\section{Acknowledgments}

Part of this work was conducted during a visit of the author G.N.T. to the Max-Planck-Institut für Plasmaphysik, Garching. The hospitality of that Institute is greatly appreciated. This work was performed within the participation of the University of Ioannina in the Association Euratom-Hellenic Republic, which is supported in part by the European Union and by the general Secretariat of Research and Technology of Greece. The views and opinions expressed herein do not necessarily reflect those of the European Commission.

\section{References}

[1] Throumoulopoulos, G. N. and Tasso, H. 2007 Phys. Plasmas 14, 122104; doi:10.1063/1.2817957.

[2] Frieman, E. and Rotenberg, M. 1960 Rev. Mod. Phys. 32, 898.

[3] Vladimirov, V. A. and Ilin, K. I. 1998 Phys. Plasmas 5, 4199. 